\title{
The POEtic Electronic Tissue and Its Role in the Emulation of Large-Scale Biologically Inspired Spiking Neural Networks Models
}

\section{J. Manuel Moreno ${ }^{\mathrm{a}}$ Yann Thoma ${ }^{\mathrm{b}}$ Eduardo Sanchez ${ }^{\mathrm{b}}$ Jan Eriksson ${ }^{c}$ Javier Iglesiasc, d Alessandro Villa ${ }^{\mathrm{d}}$}

${ }^{a}$ Technical University of Catalunya, Department of Electronic Engineering, Barcelona, Spain; ${ }^{b}$ Logic Systems Laboratory, Swiss Federal Institute of Technology Lausanne and 'Laboratory of Neuroheuristics, Information Systems Department INFORGE, University of Lausanne, Lausanne, Switzerland;

dLaboratory of Neurobiophysics, INSERM U318, University Joseph-Fourier, Grenoble, France

\section{Key Words}

Artificial tissue - Phylogenesis • Ontogenesis - Epigenesis • Learning • Spiking neural network models $\cdot$ Spike time-dependent plasticity rule $\cdot$ Programmable hardware POEtic

\section{Abstract}

One of the major obstacles found when trying to construct artefacts derived from principles observed in living beings is the lack of actual dynamic hardware with autonomous capabilities. Even if programmable devices offer the possibil-

Fax +41613061234 E-Mail karger@karger.ch www.karger.com

KARGER (c) 2006 S. Karger AG, Basel $1424-8492 / 06 / 0033-0032$ $\$ 23.50 / 0$

Accessible online at: www.karger.com/cpu
Juan Manuel Moreno Arostegui

Department of Electronic Engineering, Technical University of Catalunya, Campus Nord, Building C4, c/Jordi Girona 1-3 ES-08034 Barcelona (Spain)

Tel. +34 9340156 91, Fax +34 934016756 E-Mail moreno@eel.upc.edu
Simplexus

Nature adapts. Organisms operate using dynamic hardware programmed to respond to external and internal stimuli and so reprogram themselves to a certain degree to suit new conditions. In attempting to emulate this dynamic responsiveness, we face a major obstacle. Although we have countless programmable devices the functions of which can be changed by reconfiguring their software, a new type of electronic device that could mimic the properties of living things could adapt itself to a new environment without relying on changing the software settings. In the present paper, Manuel Moreno, Yann Thoma, Eduardo Sanchez, Jan Eriksson, Javier Iglesias, and Alessandro Villa describe just such a family of devices they term POEtic.

POEtic devices, explain the researchers, have an architecture derived from the basic organization aspects of living things: phylogenesis (evolution), ontogenesis (development) and epigenesis (learning), hence 'POE'. Phylogenesis is simply another word for evolution and reflects the mechanisms through which an organism adapts from generation to generation through natural selection as a result of environmental pressures. Ontogenesis is the development of an individual organism driven by the information contained in its genome. Development, self-replication and self-repair are examples of ontogenetic processes. Epigenesis involves the mechanisms that allow an individual to interact with its environment and involve adaptations of behaviour at the individual level. The central nervous system and the immune systems of mammals exemplify epigenetic processes.

Moreno and colleagues hoped to take inspiration from these three axes of organization to create a flexible hardware substrate - POEtic tissue - that would be able to evolve, develop, or learn. The manifestation of this aim in the form of an electronic device would, the team claims, allow science to construct an electronic tissue 
ity of modifying the functionality implemented in the device, they rely on external hardware and software elements to provide its physical configuration. In this paper we present a new family of electronic devices, called POEtic, whose architecture has been derived from the basic properties that can be extracted from the three major organization principles present in living beings: phylogenesis, ontogenesis and epigenesis. We will demonstrate that the capabilities present in these new programmable devices make them an ideal candidate for the real-time emulation of large-scale biologically inspired spiking neural network models.

Copyright @ 2006 S. Karger AG, Basel

\section{Introduction}

Even if there is a huge variability in the external features and functions associated with the living beings we can observe on earth, their organization is driven by principles that can be grouped around three main axes:

Phylogenesis: Also called evolution, it includes all the mechanisms that, driven by the pressure posed by nature, make it possible to determine the genetic information for a population of individuals that best fits a given environment.

Ontogenesis: Ontogenetic mechanisms permit the development of a single individual driven by the information contained in its genome. Apart from developmental capabilities, self-replication and self-repair (which for most living beings means healing abilities) constitute clear examples of ontogenetic processes.

Epigenesis: This includes all the mechanisms that permit a single individual to efficiently interact with its direct environment. Epigenetic mechanisms include those plasticity-oriented processes that, driven by a sensor-actuator loop, permit an organism to modify its internal structure or its behaviour in order to adapt to the specific conditions present in a given environment at any time. Examples of biological subsystems showing epigenetic principles can be found in the central nervous system of mammals and in the immune system.

Taking inspiration from these organization principles, the main goal of the POEtic project was the development of a flexible hardware substrate showing the basic features that permit living beings to show evolutionary, developmental or learning capabilities. The hardware substrate, in the form of a new electronic device, should permit the construction of electronic tissues able to solve tasks where these bio-inspired features represent a clear advantage over classical techniques.

The paper is organized as follows: in the next section we present the overall organization of the POEtic tissue, describing the details of its main constituent parts. Then we introduce the features of a new learning model for spiking neural networks models that, when used in large-scale networks, shows interesting feature extraction capabilities. Once physically implemented into the POEtic devices, it will be demonstrated that these provide an efficient prototyping instrument for neuroscience research. The paper will finish presenting the conclusions and our current work.

\section{Overall Organization of the POEtic Tissue}

The POEtic tissue is organized as a homogeneous bidimensional array of POEtic chips, each one of them being able to implement a given number of cells as required by the application to be handled. The organization of a single POEtic chip is presented in figure 1 .

From a structural point of view the organization of a POEtic chip is divided into three main sections: the environment subsystem, the organic subsystem and the system interface. The environment subsystem is in charge of managing the interactions with the environment, and also of imple- capable of solving various tasks with many clear advantages over more conventional approaches. Indeed, such a device would be an ideal candidate for a neural networks model, they explain bringing together all three axes of biology - evolution, development, and learning - in a single device.

The POEtic tissue devised by the researchers is a collection of homogeneous bi-dimensional array POEtic chips, a POEtic chip being a specially designed microprocessor that can run evolutionary algorithms and be programmable. Such a chip has three main sections: the environment subsystem, the organic subsystem and the system interface.

The environment subsystem, explain the team, controls environmental interactions as its name would suggest and so can act as the evolutionary input and processing for the POEtic tissue. The organic subsystem runs the behavioural and learning processes that are to be exhibited by the tissue. Crucially, the interface provides the means of communication between these two subsystems. Sensors and actuators connect inputs with outputs. A $3 \times 3$ array of POEtic chips is a simple POEtic tissue, but the nature of the interconnections means that such a tissue would be wholly scalable, so that any number of chips could be linked for a specific purpose. The architecture overall also means that an $\mathrm{M} \times \mathrm{N}$ array behaves as a single POEtic chip at any scale. The only difference between a single chip and a tissue, explain the researchers, is the actual physical size of the organic subsystem.

The architecture of the POEtic chip is centred around a 32-bit custom RISC processor, carrying dedicated instructions for developing evolutionary algorithms. A built-in pseudo-random number generator mimics the random mutations that occur in an organism's genome, while various advances buses connect the various components. The team explain how careful design of the system memory map the organization of the physical memory 


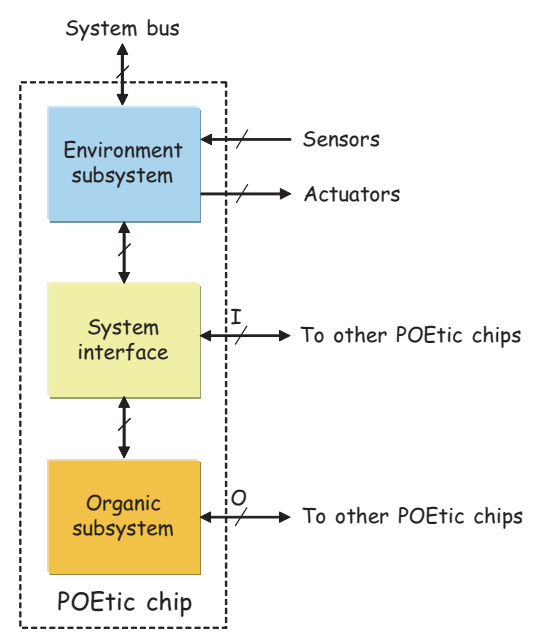

Fig. 1. Organization of a POEtic chip.

menting the phylogenetic mechanisms of the tissue. The organic subsystem manages the physical realization of the epigenetic and ontogenetic processes to be exhibited by the tissue. Finally, the system interface takes care of the efficient communication between these two subsystems. It also provides the mechanisms that permit the tissue to exhibit scalable properties.

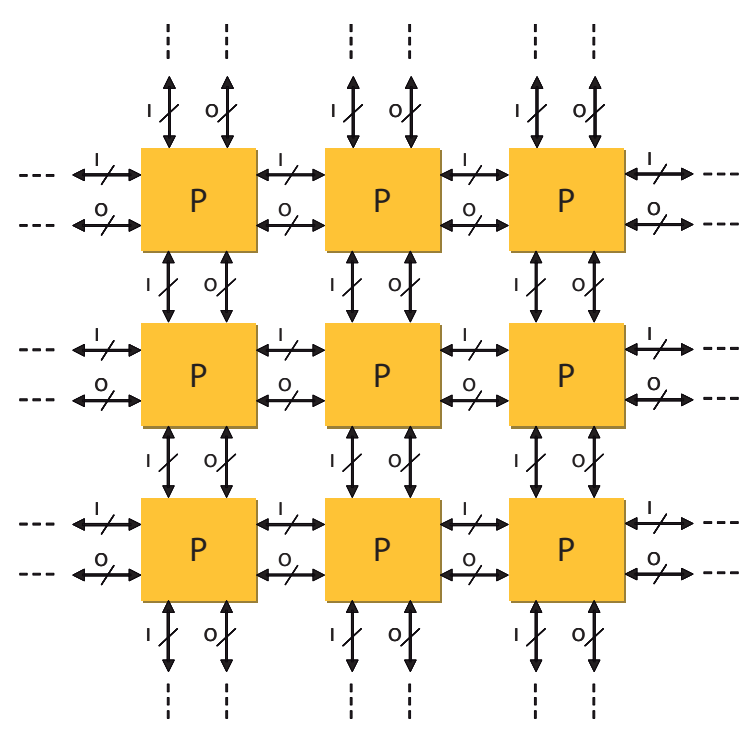

Fig. 2. Overall organization of the POEtic tissue.
The overall organization of the resulting tissue is depicted in figure 2 .

The squares in figure 2 represent POEtic chips, so that the sample tissue represented in the figure is constituted by 9 POEtic chips (the squares labelled as $\mathrm{P}$ ) organized as a $3 \times 3$ matrix. As it can be deduced from the figure, the local communication between chips is separated in two different sections. The bidirectional lines labelled as I represent those connections associated with the system interface, while the bidirectional lines labelled as 0 indicate the connections established between the organic subsystems included in every chip. As it will be explained later the connections corresponding to the system interface provide the scalability features required by the POEtic tissue, meaning that it can be constituted by as many chips as required by the actual application to be tackled. The connectivity between the organic subsystems is established at the routing plane level, and they allow for an effective communication mechanism between cells that are physically implemented in different chips. subsystems - permits the complete POEtic tissue to be managed by just one environment subsystem. The organic subsystem comprises two layers: a two-dimensional array of basic elements, called 'molecules', and a two-dimensional array of routing units, with each molecule connected to four neighbours in a regular arrangement. The interface bus then carries the signals that allow a collection of POEtic chips (a POEtic tissue) to behave as if they were a single POEtic chip. The researchers explain further that the coordinates of a given chip are not pre-programmed, but are calculated for any given configuration before a tissue is made operational. So far, the team has implemented a POEtic chip containing 144 'molecules' organized as an $8 \times 18$ array.

The first application of their POEtic tissue is to emulate a spiking, or pulsed, neural network model. This is essentially a group of interconnected artificial neurones whose connectivity adapts.

Specifically, this model consists of a leaky integrate-and-fire scheme. Here the 'weight' of connections between neurones changes depending on the timing of inputs. The team suggests that a sixteen-neurone network organized as a $4 \times 4$ POEtic array can emulate a 10,000-neuron network by running 625 cycles. This would require for real-time video processing a processor clock frequency of just $5 \mathrm{MHz}$, which the researchers explain, is far less than the actual clock frequency of the organic subsystem of their POEtic tissue. The team has tested this power using images input from an OmniVision OV5017 monochrome 384 $\times 288$ CMOS digital camera. By applying dynamic synthetic images to the neural model implemented in the tissue it was possible to verify that the learning model is able to modify the dynamics of the network so as to accommodate that present in the input stimuli.

The researchers suggest that their success with the POEtic array in mimicking a spiking neural network could serve as an 
Even if the POEtic tissue may be constructed from an arbitrary number of POEtic chips, each of them with their own functional subsystems, the system interface and the choice of the system bus makes it possible to handle the final tissue as a single POEtic chip. The only difference between a single chip and a tissue is the actual size of the organic subsystem, which in the later case is an aggregation of all the organic subsystems present in the tissue.

\subsection{The Environment Subsystem}

Figure 3 shows the internal organization of the environment subsystem.

As it can be deduced from figure 3 the architecture of the environment subsystem is structured around a specific microprocessor core. It is a 32-bit custom RISC processor, with dedicated instructions for developing evolutionary algorithms. A pseudorandom number generator is included in the ALU of the processor. The organization of the environment subsystem is organized around the AHB bus (advanced high-performance bus) corresponding to the AMBA specification [1]. Simple peripherals are placed in a separate bus section called APB (advanced periph- eral bus) that interfaces with the AHB bus through a bridge.

All the subsystems included in the POEtic tissue can be managed by the environment subsystem through a careful design of its memory map, whose structure is presented in table 1 . The numbers provided in table 1 are specified in hexadecimal format. Even if the organic subsystem of the POEtic tissue is mapped in only one memory section, in fact this section maps the organic subsystems of all the chips that are present in the tissue for a given application.

The first 25 words of the program data section are reserved for the interrupt vectors of the microprocessor. Table 2 summarizes the organization of this interrupt vector table. The content of each of these memory positions is a JUMP instruction that points to the start address of the corresponding interrupt service routine.

The priority of the interrupt sources is directly related to the value of its associated interrupt vector, being thus the internal interrupt 0 , the interrupt source with the highest priority.

The communication unit included in the environment subsystem makes it pos- excellent development and experimentation instrument for neuroscientists.

Another important application domain for the technology developed in the POEtic tissue is related to self-repairing hardware. The dynamic routing mechanisms supported by the organic subsystem permit the implementation of self-replication and self-repair methods. These allow for the physical realization of robust systems for safe critical applications. Such capabilities open up the possibility of creating autonomous systems able to operate in remote or hostile environments where close human supervision is not possible.

Finally, the technology developed for the POEtic tissue has also been used to create an interactive artistic installation, called POEtic-Cubes. In this installation a person or a group of people can interact with nine autonomous robots controlled by the POEtic chips. By interacting with the robots the people can experience the main steps that gave rise to life on earth, including cellular replication, cellular differentiation and phenotype expression.

David Bradley of Sciencebase.com

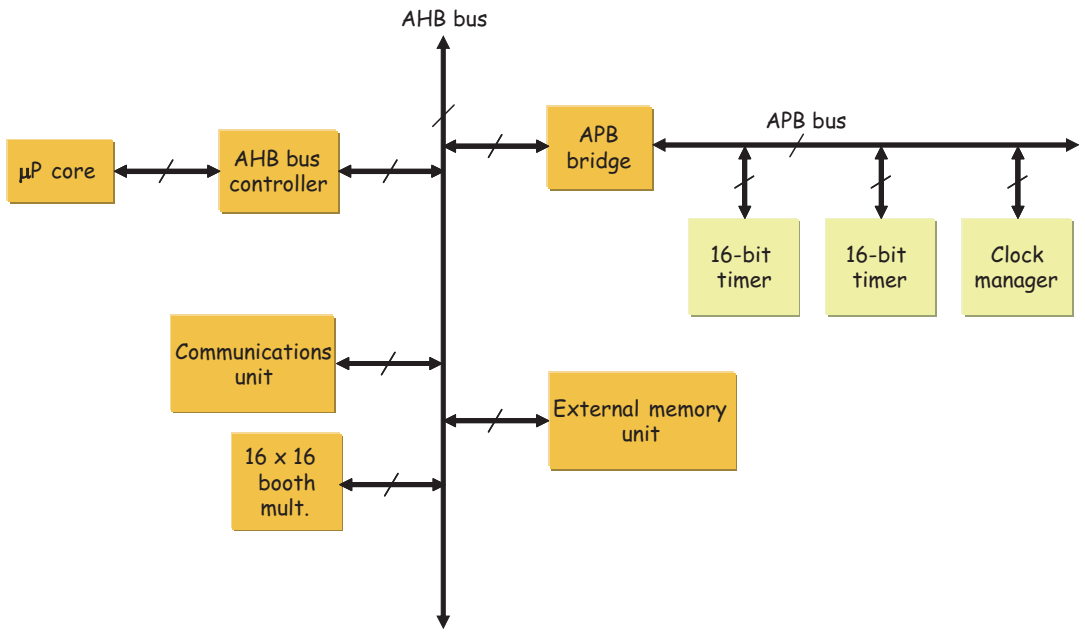

Fig. 3. Internal organization of the environment subsystem. 
Table 1. Memory map organization of the POEtic tissue

\begin{tabular}{lll}
\hline Section & Start address & End address \\
\hline Program & 0x0000_0000 & 0x3FFF_FFFF \\
Data & 0x4000_0000 & 0x7FFF_FFFF \\
Multiplier & 0xC000_0000 & 0xC000_0003 \\
Communications unit & 0xD000_0000 & 0xD000_0150 \\
Timers & 0xE000_0000 & 0xE000_0007 \\
Clock manager & 0xE000_0008 & 0xE000_000F \\
Organic subsystem & 0xF000_0000 & 0xFFFF_FFFF
\end{tabular}

sible to implement an 8-bit bidirectional port, two UARTs, one SPI interface and one I2C interface. The functionality of these interfaces can be programmed by the user to match the requirements of a given application.

The clock manager unit has been added to the environment subsystem of the POEtic tissue in order to facilitate the hardware debugging procedures for the functionality implemented in the organic subsystem. This unit permits to generate a clock signal for the organic subsystem whose frequency is divided with respect to that associated with the system clock. Furthermore, if desired, this unit makes it also possible to stop the clock signal provided to the organic subsystem after a specified number of clock cycles (from 1 to 65,535). This feature allows for advancing the state of the organic subsystem edge-by-edge and then observing it (note that the environment subsystem has access through the system interface to the configuration and state of the organic subsystem).

From an architectural point of view the organization of the external memory unit of the environment subsystem is divided into three main parts: the boot ROM, the program ROM and the data RAM.

The presence of a boot ROM section permits the user to load upon a power-up sequence, a program that may be transferred to the microprocessor using any one of the peripherals included in the communications unit. This means that the physical architecture of the memory unit of the microprocessor has two possible configurations, as depicted in figure 4 .

The organization depicted in figure $4 \mathrm{a}$ corresponds to a situation where the program to be executed by the microprocessor is fixed and already stored in a ROM.In this case after the power-up sequence the microprocessor starts executing directly from this memory section. Figure $4 \mathrm{~b}$ shows an organization corresponding to a case where there is just a boot loader program stored in a boot ROM that takes care of capturing through one of the peripherals included in the communications unit the actual program to be executed by the microprocessor. This program is stored in the program ROM section that is physically implemented by means of a Flash or a SRAM unit. In order to permit the microprocessor to physically write the program ROM section during this boot sequence the memory map is slightly changed, so that the program ROM section is mapped in the memory area starting at address 0x6000_0000.

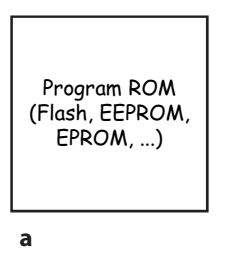

Table 2. Organization of the interrupt vector table of the microprocessor

\begin{tabular}{ll}
\hline Interrupt source & Interrupt vector \\
\hline Main program & 0x0000_0000 \\
Timer 0 & 0x0000_0001 \\
Timer 1 & 0x0000_0002 \\
Multiplier & 0x0000_0003 \\
Clock manager & 0x0000_0004 \\
UART 0 TX & 0x0000_0008 \\
UART 0 RX & 0x0000_0009 \\
UART 1 TX & 0x0000_000A \\
UART 1 RX & 0x0000_000B \\
I2C & 0x0000_000B \\
SPI & 0x0000_000C \\
Parallel port & 0x0000_000D \\
External interrupt 1 & 0x0000_0010 \\
External interrupt 0 & 0x0000_0018
\end{tabular}

\subsection{The Organic Subsystem}

The organic subsystem is made up of 2 layers, as depicted in figure 5: a two-dimensional array of basic elements, called molecules, and a two-dimensional array of routing units. Each molecule is connected to its four neighbours in a regular structure. Mainly containing a 16-bit look-up table (LUT) and a flip-flop (DFF), it has the capability of accessing the routing layer that is used for intercellular communication. This second layer implements a dynamic routing algorithm allowing the creation of data paths between cells at runtime.

A molecule is the smallest programmable element of the POEtic tissue. It is

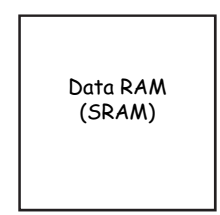

Fig. 4. Physical architecture of the external memory unit of the environment subsystem. 


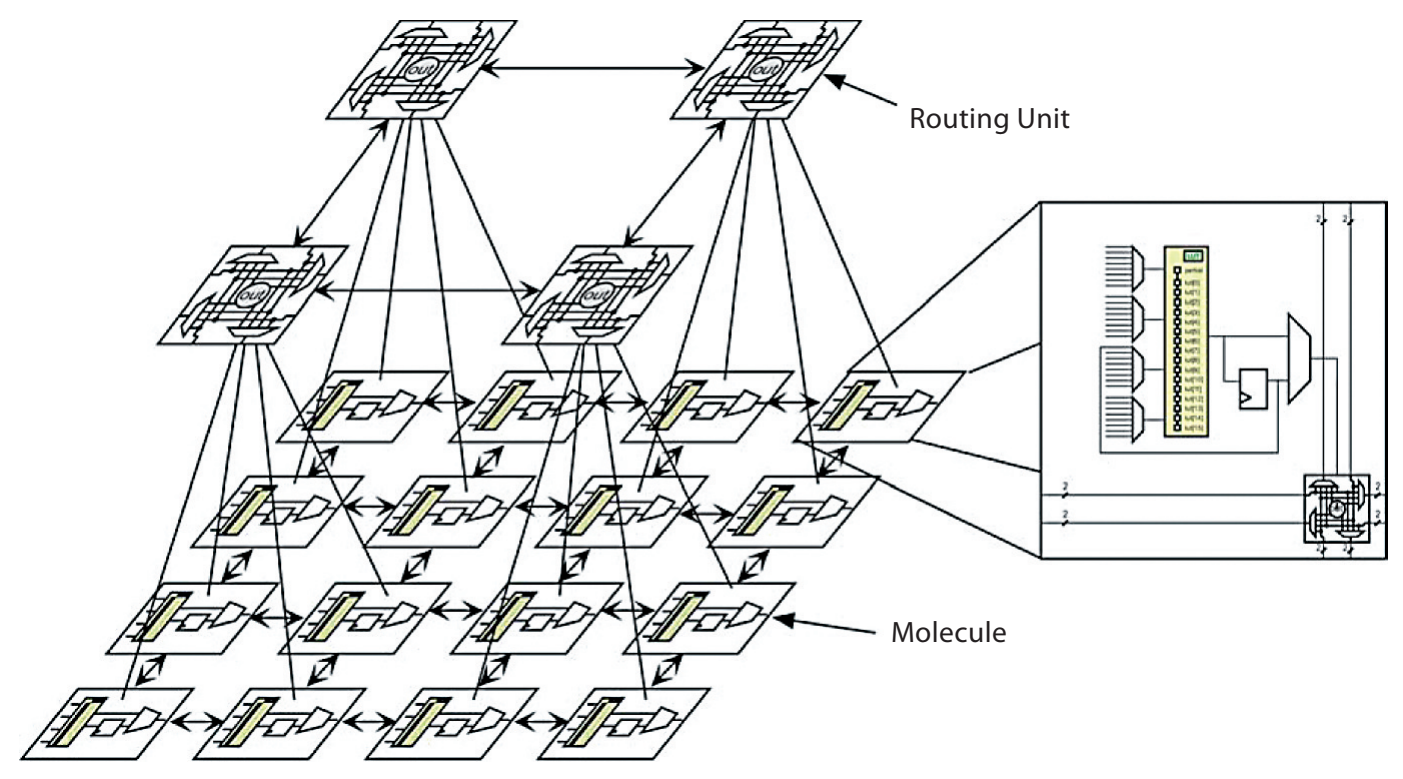

Fig. 5. Organization of the organic subsystem.

mainly composed of a flip-flop (DFF), and a 16-bit LUT (fig. 5). Eight modes of operation are supplied to ease the development of applications that need cellular systems and/or growth and self-repair. The LUT is composed of a 16-bit shift register that can be split in two, used as a shift register, or as a normal LUT.

A molecule has eight different operational modes, to speed up some operations, and to use the routing plane. The functional modes provided for the molecules are the following:

- In 4-LUT mode, the 16-bit LUT supplies an output, depending on its four inputs.

- In 3-LUT mode, the LUT is split into two 8-bit LUTs, both supplying a result depending on three inputs. The first result can go through the flip-flop, and is the first output. The second one can be used as a second output, and is directly sent to the south neighbour (can serve as carry-in parallel operations).

- In Comm mode, the LUT is split into one 8-bit LUT, and one 8-bit shift register. This mode could be used to compare a serial input data with a data stored in the 8bit shift register.
- In Shift Memory mode, the 16 bits are used as a shift register, in order to store data, for example a genome. One input controls the shift, and another one is the input of the shift memory.

- In Input mode, the molecule is a cellular input, connected to the intercellular routing plane. One input is used to enable the communication. When inactive, the molecule can accept a new connection, but will not initiate a connection. When active, a routing process will be launched at least until this input connects to its source. A second input selects the routing mode of the entire POEtic tissue.

- In Output mode, the molecule is a cellular output, connected to the intercellular routing plane. One input is used to enable the communication. As in Input mode, when inactive, the molecule can accept a new connection, but will not initiate a connection. When active, a routing process will be launched at least until this output connects to one target. Another input supplies the value sent to the routing plane, as to another cell.

- In Trigger mode, the 16-bit shift register should contain ' 000 ... 01' for a 16-bit address system. It is used by the routing plane to synchronize the address decoding during the routing process. One input is a circuit enable that can disable every DFF in the tissue, and another one can reset the routing plane, and so start a new routing. - In Configure mode, the molecule can partially configure its neighbourhood. One input is the configuration control signal, and another one is the configuration shifting to the neighbours.

Long distance intermolecular communication is possible by way of switch boxes. Each switch box consists of eight input lines (two from each cardinal direction) and eight corresponding output lines, and is implemented with eight input multiplexers. Two outputs are sent into each of the four neighbours of the molecule, as shown in figure 6.

Each output line can be connected to one of the six input lines from the other cardinal directions (no u-turns allowed) or to one of two possible outputs of the molecules (the output or the inverted output).

A molecule is defined by 75 configuration bits. They are configured by loading 


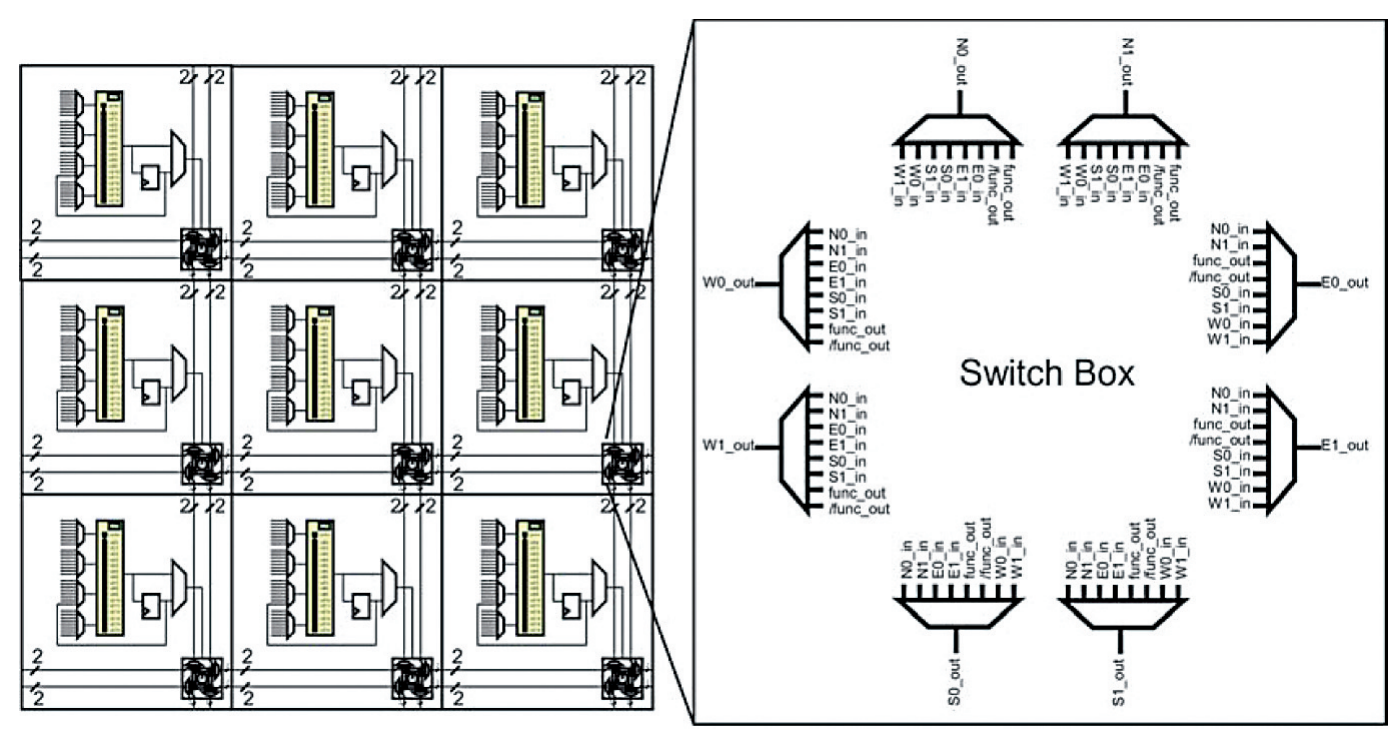

Fig. 6. Nine molecules, connected through their switchboxes, and detailed view of a switchbox.

them in parallel, from the microcontroller. A partial reconfiguration is also possible, a molecule being able to shift configuration bits of its neighbourhood. Actually, when shifting, 76 bits are used, as the value of the flip-flop has to be in the configuration chain, in order to be able to retrieve its value.

The configuration system of the molecules can be seen as a shift register of 76 bits split into 5 blocks: the LUT, the selection of the LUT input, the switch box, the mode of operation, and an extra block for all other configuration bits. Each block contains, together with its configuration, one bit indicating, in case of a reconfiguration coming from a neighbour, whether the block has to be bypassed (as shown in fig. 7). This bit can only be loaded from the microprocessor, and remains stable during the entire lifetime of the organism.

The special configure mode allows a molecule to partially reconfigure its neighbourhood. It sends bits coming from another molecule to the configuration of one of its neighbours. By chaining the configurations of neighbouring molecules, it is possible to modify multiple molecules at the same time, allowing, for example, the synaptic weights in a neuron to be changed.

Three configuration bits are used to define the possible origin of a partial reconfiguration: two bits for selecting the origin, and one bit that enables the partial configuration. In case a neighbour tries to partially reconfigure the molecule, if this config_partial_enable bit is set to ' 1 ', then the molecule is partially reconfigured, and it tries to partially reconfigure its neighbours, by chaining the output of the configuration stream. If the config_partial_ enable bit is set to ' 0 ', then no partial reconfiguration is executed, and no signal is sent to the neighbours.

This partial reconfiguration allows for instance to use the configuration bits of a molecule to store information. A maximum of 54 bits can be stored in only one molecule, allowing for efficiently implementing genome storage. By modifying the LUT content, a cell can also modify its behaviour, which is a useful feature for evolvable hardware.

The second plane of the organism subsystem implements a dynamic routing al- gorithm to allow the circuit to create paths between different parts of the molecular array. The possibility of having a pseudostatic routing has also been added, to ease the development of applications that only need local connections between cells.

The dynamic routing system is designed to automatically connect the cells' inputs and outputs. Each output of a cell has a unique identifier at the organism level. For each of its inputs, the cell stores the identifier of the source from which it needs information. A non-connected input (target) or output (source) can initiate the creation of a path by broadcasting its identifier, in case of an output, or the identifier of its source, in case of an input. The path is then created using a parallel implementation of the breadth-first search algorithm. When all paths have been created, the organism can start operation, and execute its task, until a new routing is launched, for example after a cell addition or a cellular self-repair.

Our approach has many advantages, compared to a static routing process. First of all, a software implementation of a shortest path algorithm, such as that of Di- 


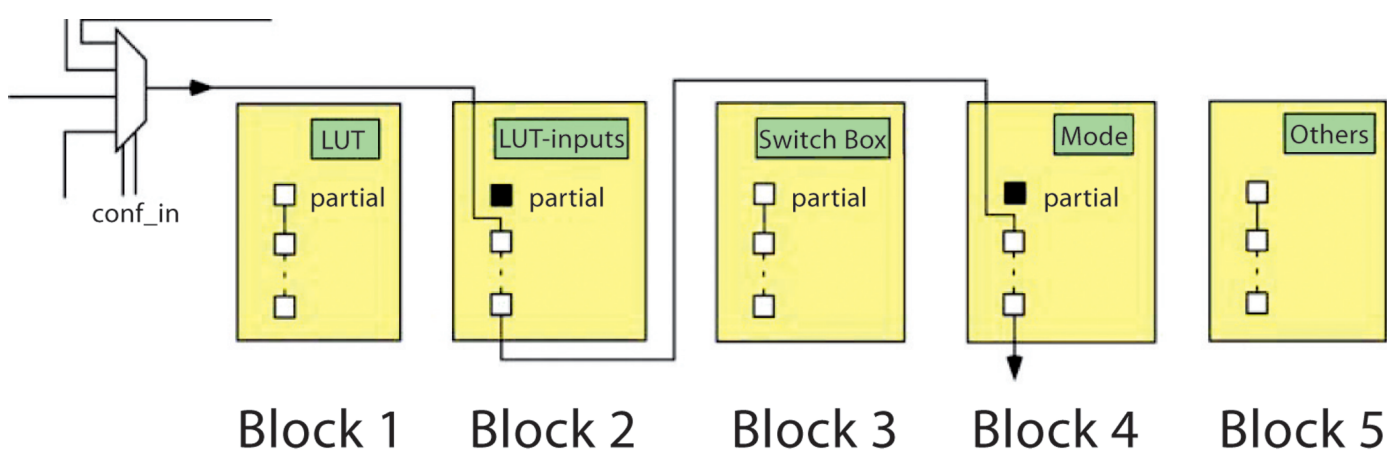

Fig. 7. Organization of the configuration bits for partial reconfiguration.

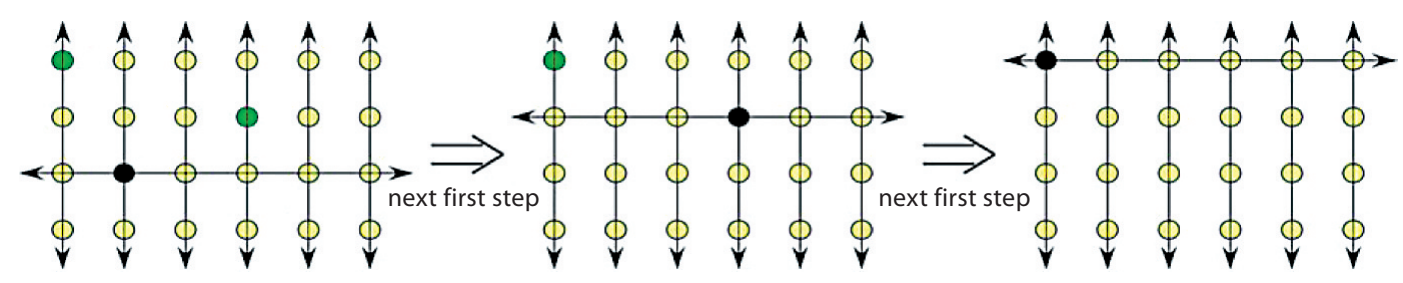

Fig. 8. Three consecutive steps of the routing algorithm. The black routing unit will be the master, and therefore will perform its routing.

jkstra [2], is very time-consuming for a processor, while our parallel implementation requires a very small number of clock cycles to finalize a path. Second, when a new cell is created it can start a routing process, without the need of recalculating all paths already created. Third, a cell has the possibility of restarting the routing process of the entire organism, if needed (for instance after self-repair). Finally, our approach is totally distributed, without any global control over the routing process, so that the algorithm can work without the need of the central microprocessor.

Every routing unit is composed of a switch box and a finite state machine. The switch box contains five multiplexers that can select the value sent to each of the four neighbours, and to the molecules underneath. The state machine is responsible for correctly configuring the multiplexers, and implements the distributed routing algorithm, by communicating with the other routing units.
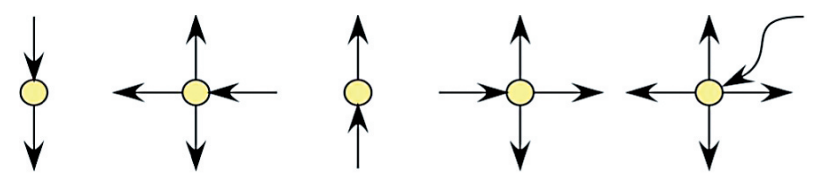

Fig. 9. The propagation direction of the address: north $\rightarrow$ south | east $\rightarrow$ south, west, and north | south $\rightarrow$ north | west $\rightarrow$ north, east, and south | routing unit $\rightarrow$ north, east, south, and west.

The routing algorithm is executed in four phases:

\section{Phase 1: Finding a Master}

In this phase, every target or source that wants to be but is not connected to its correspondent partner tries to become master of the routing process. A simple priority mechanism chooses the most bottom-left routing unit to be the master, as shown in figure 8. Note that there is no global control for this priority, with every routing unit knowing whether or not it is the master. This phase is over in one clock cycle, as the propagation of signals is combinational.

\section{Phase 2: Broadcasting the Address}

Once a master has been selected, it sends its address in case of a source, or the address of the needed source in case of a target. It is sent serially, in n clock cycles, where $n$ is the size of the address. The same path as in the first phase is used to broadcast the address, as shown in figure 9. 
Every routing unit, except the one that sends the address, compares the incoming value with its own address (stored in the molecule underneath). At the end of this phase, that is, after $\mathrm{n}$ clock cycles, each routing unit knows if it is involved in this path. In practice, there has to be one and only one source, and at least one target.

\section{Phase 3: Eliminating Sources and}

Targets

In some situations, a source should start a routing process, for instance, in a developmental process. In such a process, it would be useful to have many sources and targets with the same ID. So at this stage, it is possible that there is more than one source involved in the routing process. In order to avoid multiple sources, in this phase that lasts only one clock cycle, if a source is at the origin of the routing process, it sends a signal to every other routing unit, to let them know a source is at the origin. Then every other source with the same ID disables its participation in the current process, and during the next phase, the source will connect to the nearest target. The same disable is performed in case a target launched the routing process. Every target that is not the master disables its participation to the current process, to ensure that the target that started the process will be the only one connected to a source. In this case, the nearest source will be connected to this target.

Phase 4: Building the Shortest Path

The last phase, largely inspired by Moreno Arostegui [3], creates a shortest path between the selected source and the selected targets. An example involving 8 sources and 8 targets is shown in figure 10 , for a densely connected network.

A parallel implementation of the breadth-first search algorithm allows the routing units to find the shortest path between a source and many targets. Starting from the source, an expansion process tries to find targets. When one is reached,

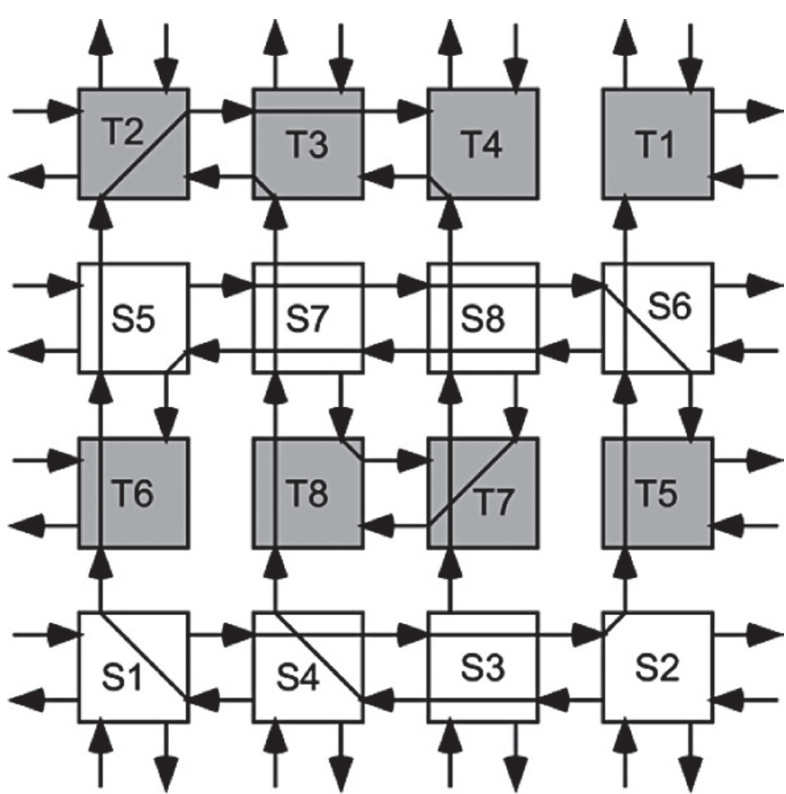

Fig. 10. Test case with a densely connected network.
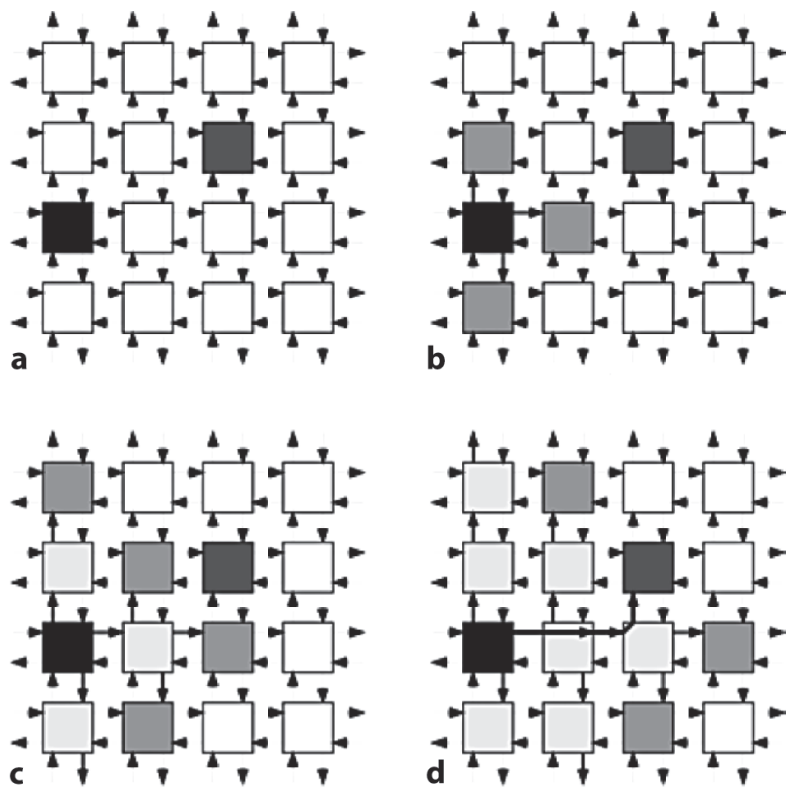

Fig. 11. Step one (a), two (b), three (c) and four (d) of the path construction process between the source placed in column 1, row 2 and target cell placed in column 3, row 3 .

the path is fixed, and all the routing resources used for the path will not be available for the next successive iterations of the algorithm.
Figure 11 shows the development of the algorithm, building a path between a source placed in column 1, row 2 and a target cell placed in column 3 , row 3 . After 3 clock cycles of expansion, the target is 


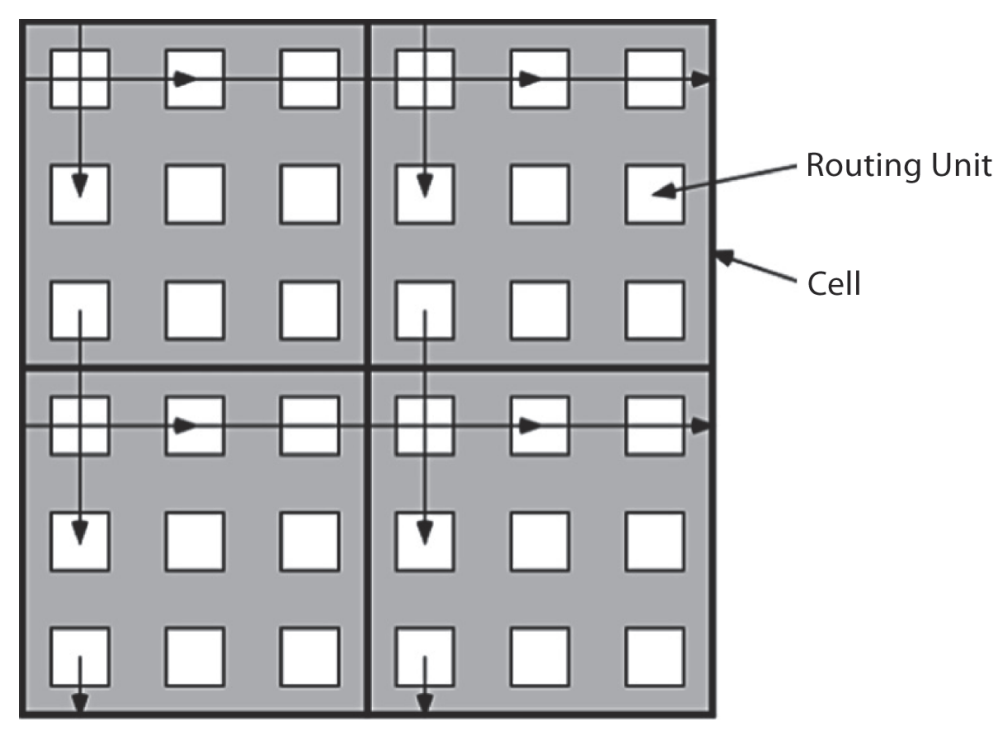

Fig. 12. A pseudostatic communication scheme between four cells.

reached, and the path is fixed, prohibiting the use of the same path for a successive routing.

Based on addresses, the dynamic routing presented above is very flexible. However, for some applications, this flexibility can become a disadvantage, for example if we only need local communications between cells like a 4-neighbourhood.

A second mode of routing has been added for this purpose. A flip-flop in the tissue can be configured by the molecules to choose the mode to use for a specific application. The pseudostatic mode uses the fact that every switch box is pass-through after a hardware reset. When in pseudostatic mode, the routing units that are connected to input or output molecules only shift the content of the molecule LUT into the configuration of the switchbox. By this way, in 16 clock cycles, the intercellular routing is completed, and the circuit can start its task. The only limitation is that a path between two cells can only be a vertical or a horizontal one, without more complex possibilities (fig. 12).

\subsection{The System Interface}

As it has been mentioned previously, the system interface of the POEtic tissue plays a major role in allowing for its scalability features. This means that the physical size of the tissue can be accommodated to the actual needs of a given application without posing specific constraints either on the system architecture or in the connectivity pattern among the POEtic chips that constitute the tissue.

The POEtic tissue, as it was presented in figure 1, can be constructed as a bidimensional array constituted by POEtic chips. The connectivity between these chips, as depicted in this figure, is based on two different buses, named organic (0) and interface (I) buses. The signals that constitute the organic bus allow the organic subsystems present in every POEtic chip to communicate (at a cellular level).

The interface bus carries those signals that permit to handle the collection of POEtic chips as a single tissue, so that from a user point of view the tissue has

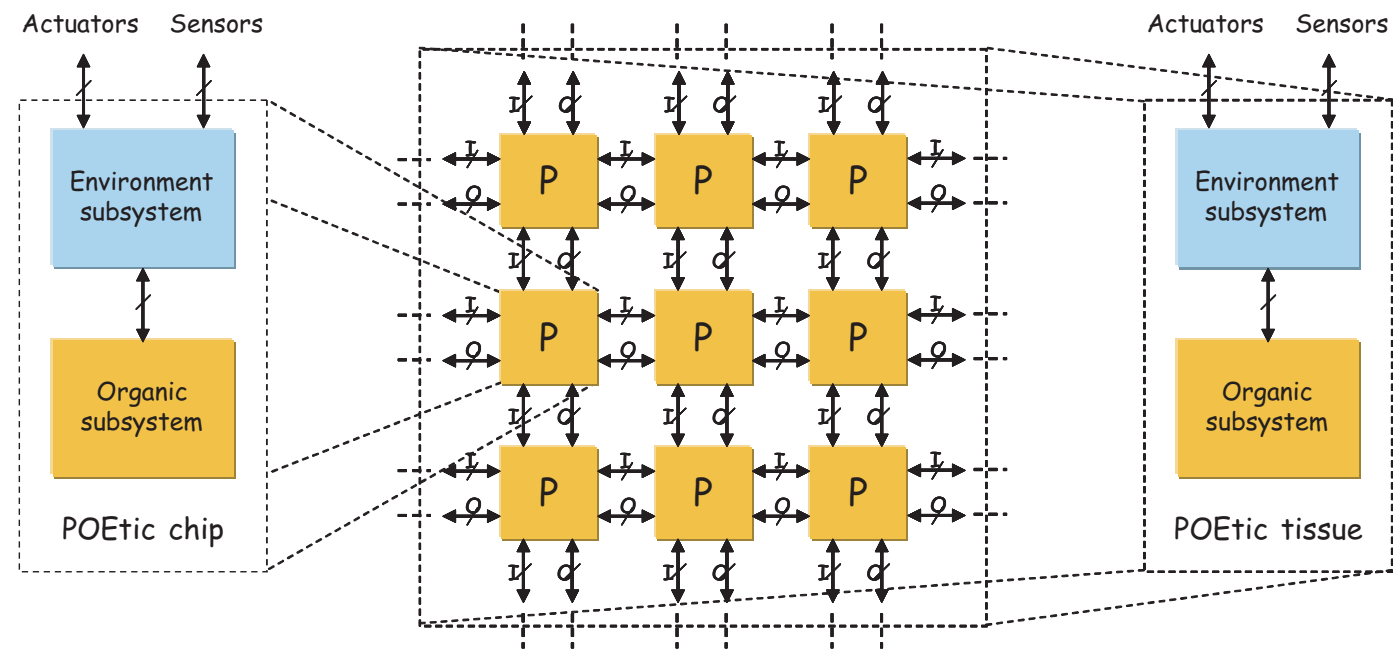

Fig. 13. Scalability properties of the POEtic tissue. 


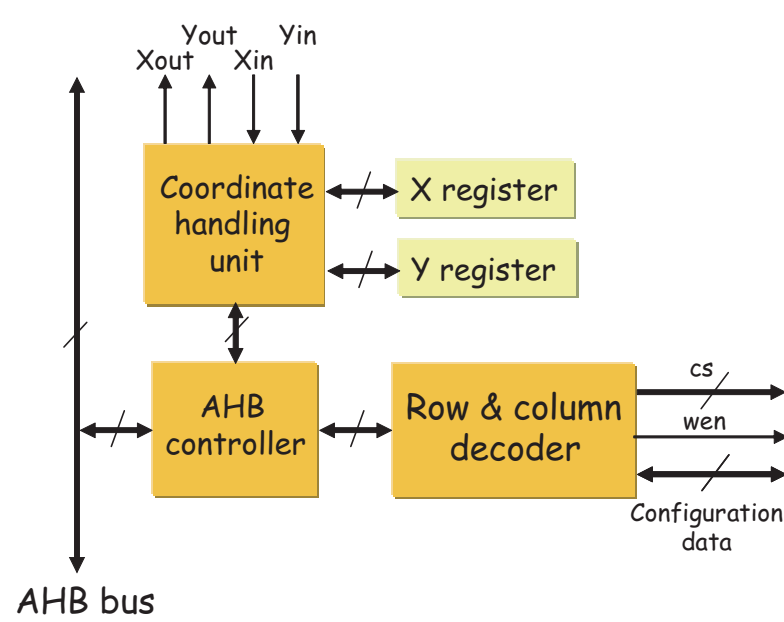

Fig. 14. Internal organization of the system interface.

only one environment subsystem and one organic subsystem. This is represented in figure 13.

Regarding the scalability of the environment subsystem, even if every POEtic chip contains a single environment subsystem, only one of them will be active in the tissue. This is accomplished by a specific signal present in every POEtic chip, called master, that indicates (when set to a value ' 0 ') that the environment subsystem of a specific chip will be managing the complete tissue.

The 68 signals ( 32 data lines, 32 address lines, sahbi_hsel, sahbi_hready, sahbi_ hwrite and sahbo_hready) that constitute the AHB bus used for the POEtic tissue are connected to all the POEtic chips. This means that the chip identified as a master of the system can access the resources present in any other chip. A specific chip is identified within the array using Cartesian coordinates that correspond to the physical position of the chip in the array. This means that a chip with coordinates $(\mathrm{X}, \mathrm{Y})$ is placed in column $\mathrm{X}$ and row $\mathrm{Y}$ within the array.

The coordinates of a given chip are not preprogrammed, but are calculated for a given array configuration during a coordi- nate propagation phase that should be performed before the tissue is operational. For this purpose every POEtic chip has two inputs, named Xin and Yin, and two outputs, Xout and Yout. The Xin input of a given chip is connected to the Xout output of the chip placed in the same row and in the previous column within the array. The Yin input of a given chip is connected to the Yout output of the chip placed in the same column and in the previous row within the array.

Every POEtic chip receives in serial mode its X coordinate through its Xin input and its $\mathrm{Y}$ coordinate through its Yin input. The coordinates are received in serial mode, so that by default the Xin and Yin inputs are in idle state (i.e., with a value ' 0 '), and after one of these input is set to value ' 1 ' the POEtic chip should recognize that during the next 4 (in the current version of the POEtic chip the $\mathrm{X}$ and $\mathrm{Y}$ coordinates are 4-bit wide, but this can be easily extended to any desired size) cycles its $\mathrm{X}$ or $\mathrm{Y}$ coordinate will be received through the corresponding input. Once a given chip has received its $\mathrm{X}$ and $\mathrm{Y}$ coordinates it calculates and sends the coordinates for its direct neighbours. The coordinate propagation process is started by the chip whose environment subsystem has been identified as a master. The coordinate propagation process is started when the microprocessor included in the environment subsystem of the master chip performs a write cycle on the address 0xF000_0004 (as it was indicated in table 1 , the organic subsystem is mapped in the memory space ranging from $0 x F 000$ 0000 to 0xFFFF_FFFF).

Once all the chips have got their actual coordinates within the POEtic tissue it is quite simple for the environment subsystem to access the organic subsystem present in any chip. In order to access (either in read or write mode) the configuration of a specific molecule present in a POEtic chip placed at coordinates $(\mathrm{X}, \mathrm{Y})$ the environment subsystem should perform a read or write access to the memory position 0xF00X_YABC, where:

- $\mathrm{X}$ : Row where the POEtic chip is placed.

- Y: Column where the POEtic chip is placed.

- $\mathrm{A}(3: 0) \mathrm{B}(3: 0) \mathrm{C}(3: 2)$ : These 10 bits indicate the address of the molecule within the chip. One POEtic chip contains 144 molecules, and their mapping ranges from 0x002 to 0x091. 


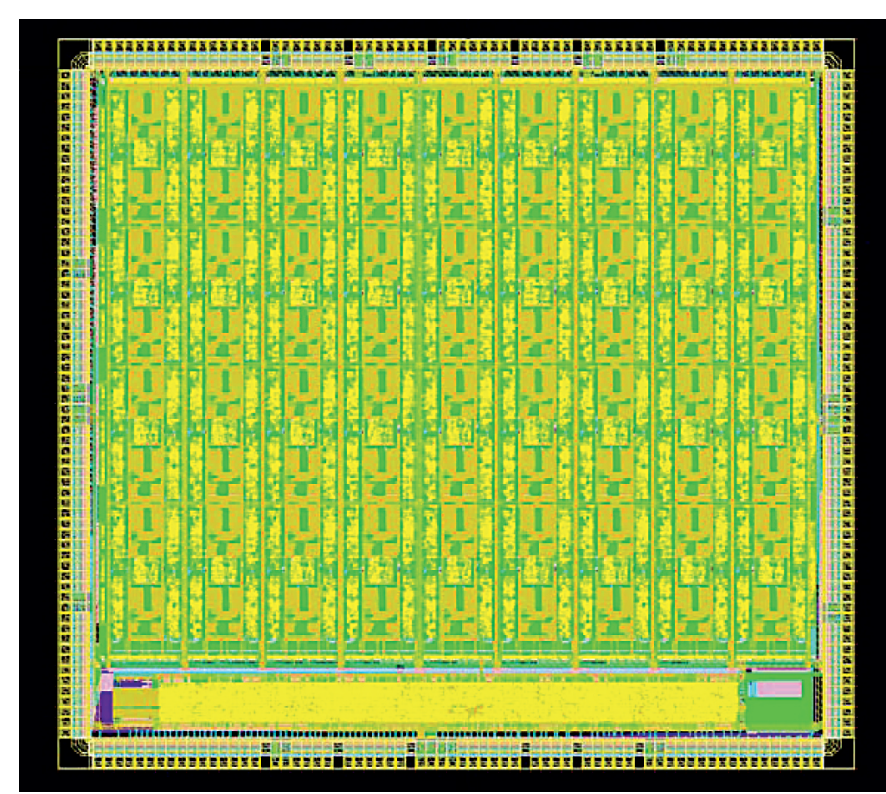

Fig. 15. Layout of the POEtic chip.

- $\mathbf{C}(1: 0)$ : These 2 bits indicate which one of the 3 configuration words of the molecule are to be read or written. A value ' 01 ' implies the activation of the csl signal, a value ' 10 ' implies the activation of the cs2 signal, while a value ' 11 ' implies the activation of the cs3 signal.

Bearing this in mind, the final organization of the system interface included in every POEtic chip is that depicted in figure 14.

The wen signal depicted in this figure indicates whether the access to the configuration of a given molecule is in read or write mode. The bidirectional configuration data bus is in fact constituted by two independent 32-bit buses, one for read access and the other for write access.

\subsection{Physical Implementation}

The POEtic chip has been implemented and fabricated as an ASIC of $54 \mathrm{~mm}^{2}$ using a $0.35-\mu \mathrm{m}$ CMOS process. The chip, whose layout is depicted in figure 15 , contains 144 molecules organized as an $8 \times 18$ array and the complete environment subsystem explained in previous sections.

\section{Emulation of Large-Scale Spiking Neural Network Models}

The spiking neural network model considered in our approach is that presented in Eriksson et al. [4]. This model outperforms previous approaches for implementing spike time-dependent plasticity-like learning methods when dealing with dynamic input stimuli.

Basically, this model consists of a leaky Integrate-And-Fire scheme, in which synapses can change their weights depending on the time difference between spikes. The outputs of the synapses are added until their result $\mathrm{V}_{\mathrm{i}}(\mathrm{t})$ overcomes a certain threshold $\theta$. Then a spike is produced, and the membrane value is reset.

The simplified equation of the membrane value is:

$$
V_{i}(t+1)= \begin{cases}0 & \text { when } S_{i}(t)=1 \\ k_{\text {mem }} \cdot V_{i}(t)+\sum J_{i j}(t) & \text { when } S_{i}(t)=0\end{cases}
$$

where $\mathrm{k}_{\mathrm{mem}}=\exp \left(-\Delta \mathrm{t} / \tau_{\mathrm{mem}}\right), \mathrm{V}_{\mathrm{i}}(\mathrm{t})$ is the value of the membrane, $\mathrm{J}_{\mathrm{ij}}$ is the output of each synapse and $S_{i}(t)$ is the variable which shows when there is a spike.
The goal of the synapse is to convert the spikes received from other neurons in proper inputs for the membrane. When there is a spike in the presynaptic neuron, the actual value of the output $\mathrm{J}_{\mathrm{ij}}$ is added to the weight of the synapse multiplied by its activation variable. But if there is no presynaptic spike, then the output $\mathrm{J}_{\mathrm{ij}}$ is decremented by the factor $\mathrm{k}_{\text {syn. }}$. The output $\mathrm{J}$ of the synapse ij is ruled by:

$J_{i j}(t+1)= \begin{cases}J_{i j}(t)+\left(w_{R i R j} \cdot A_{R i R j}(t)\right) & \text { when } S_{j}(t)=1 \\ k_{\text {syn }} \cdot J_{i j}(t) & \text { when } S_{j}(t)=0\end{cases}$

where $\mathrm{j}$ is the projecting neuron and $\mathrm{i}$ is the actual neuron. $R$ is the type of the neuron: excitatory or inhibitory, $\mathrm{A}$ is the activation variable which controls the strength of the synapse, and $\mathrm{k}_{\text {syn }}$ is the kinetic reduction factor of the synapse. If the actual neuron is inhibitory, this synaptic kinetic factor will reset the output of the synapse after a time step, but if the actual neuron is excitatory, it will depend on the projecting neuron. If the projecting neuron is excitatory the synaptic time constant will be higher than if it is inhibitory. The weight of each synapse also depends on the type of neuron it connects. If the synapse connects two inhibitory neurons, the weight will always be null, so an inhibitory cell cannot influence another inhibitory cell. If a synapse is connecting two excitatory neurons, it is assigned a small weight value. This value is higher for synapses connecting an excitatory neuron to an inhibitory one, and it takes its maximum value when an inhibitory synapse is connected to an excitatory cell.

In order to strengthen or weaken the excitatory-excitatory synapses, the variable A will change depending on an internal variable called $\mathrm{L}_{\mathrm{ij}}$ which is ruled by:

$$
\begin{aligned}
& \mathrm{L}_{\mathrm{ij}}(\mathrm{t}+1)=\mathrm{k}_{\mathrm{act}}{ }^{*} \mathrm{~L}_{\mathrm{ij}}(\mathrm{t})+\left(\mathrm{YD}_{\mathrm{j}}(\mathrm{t}) * \mathrm{~S}_{\mathrm{i}}(\mathrm{t})\right)- \\
& \left(\mathrm{YD}_{\mathrm{i}}(\mathrm{t}) * \mathrm{~S}_{\mathrm{j}}(\mathrm{t})\right)
\end{aligned}
$$

where $k_{a c t}$ is the kinetic activity factor, which is the same for all the synapses. 
YD is the learning variable that measures, with its decay, the time separation between a presynaptic spike and a postsynaptic spike. When there is a spike, YD will have its maximum value in the next time step, but when there is not, its value will be decremented by the kinetic factor $\mathrm{k}_{\text {learn }}$, which is the same for all synapses.

When a presynaptic spike occurs just before a postsynaptic spike, then the variable $\mathrm{L}_{\mathrm{ij}}$ increases and the synapse strengthens. This means it reinforces the effect of a presynaptic spike in the soma. But when a presynaptic spike occurs just after a postsynaptic spike, the variable $\mathrm{L}_{\mathrm{ij}}$ decreases, the synapse weakens and the effect of a presynaptic spike in the soma will decrease. For other kinds of synapses, the activation variable is always equal to 1 .

Regarding the network configuration, $80 \%$ of the neurons are excitatory, while the remaining $20 \%$ are inhibitory. Each cell makes connections with other neurons within a $5 \times 5$ neighbourhood, i.e. 24 neurons. Figure 16 represents this connectivity pattern.

The parameters that govern the functionality of the neuron block are:

- The membrane path has a resolution of 12 bits, with a range [-2,048, 2,047], and the threshold is kept fixed to +640 .

- The membrane decay function has a time constant value of $\tau=20$.

- The refractory time is set to 1 .

The decay block will be used both in the learning and synapse blocks. Its goal is to have a logarithmic decay of the input; it is obtained with a subtraction and controlling the time when it is done depend-

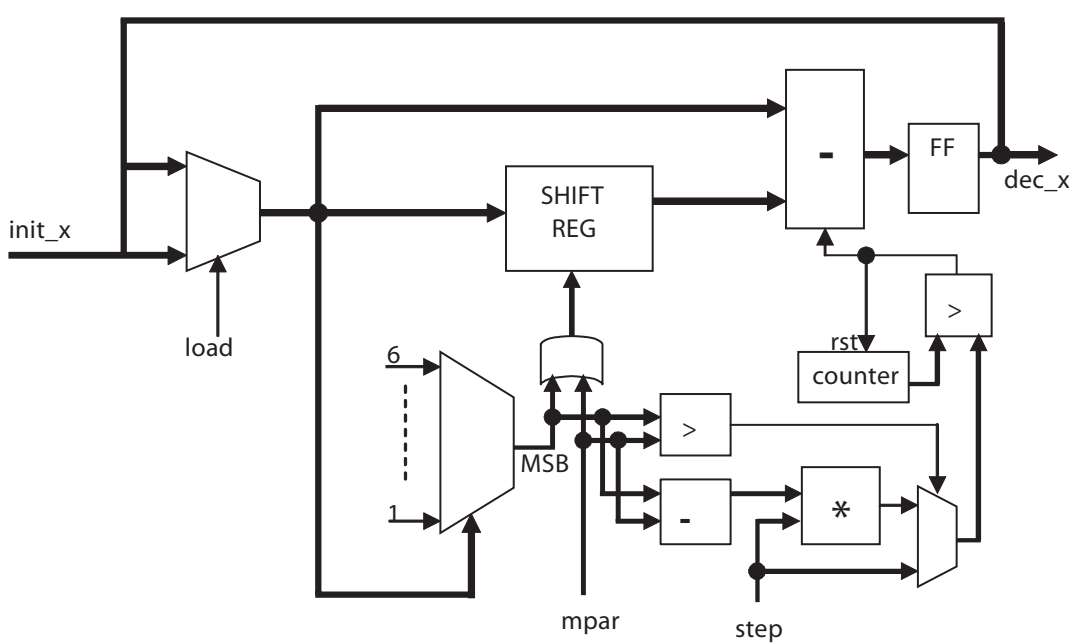

Fig. 17. Block diagram of the decay block.

ing on the input value. Taking into account that this block is used in many parts of the design, the decayed variable has been called $\mathrm{x}$.

The block diagram is represented in figure 17. First of all, a new value of $x$ should be obtained. It will be the input of a shift register which is controlled by the most significant bit of $\mathrm{x}$ and the external parameter mpar.

The output of this shift register will be subtracted from the original value of $\mathrm{x}$. This operation will be done when indicated by the time control. The time control is done with the value of a counter that is compared with the result of choosing between the external value step or the multiplication of (MSB - mpar) by step. The decay variable $\tau$ depends on the input parameters mpar and step.
The learning block 'measures' the time difference between a spike in the projecting neuron ( $\mathrm{j}$ ) and the actual neuron (i). Depending on these time differences and the types of the neurons, the synapse will be more or less active.

When a spike is produced in the projecting neuron, the variable YD loads its maximum value and starts to decay slowly. Then, if the actual neuron spikes, the value of $\mathrm{YD}_{\mathrm{j}}$ is added to the decayed value of the $\mathrm{L}$ variable. On the other hand, if a spike is produced first in the actual neuron and after in the projecting neuron, the value of $\mathrm{YD}_{\mathrm{i}}$ is subtracted from the decayed value of the $\mathrm{L}$ variable.

When the L variable overcomes a certain threshold (L_th), positive or negative, the activation variable (A) increases or decreases, respectively, unless it is already in
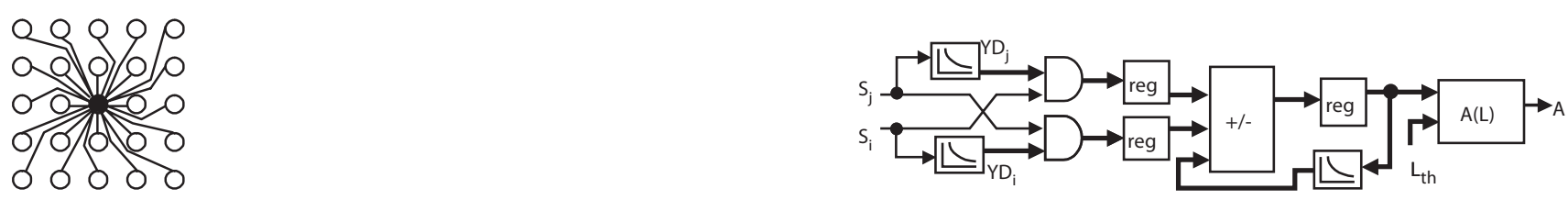

Fig. 16. Connectivity of a single neuron.

Fig. 18. Organization of the learning block. 


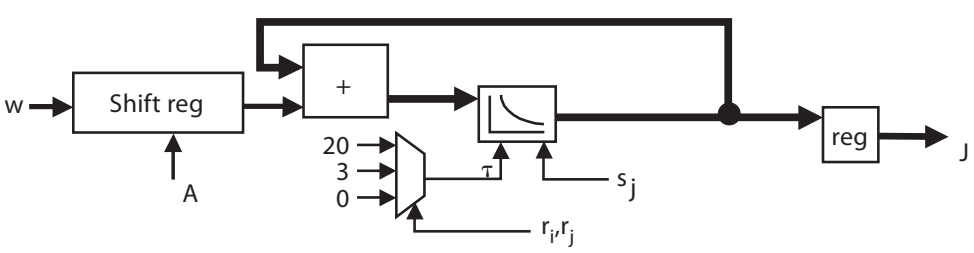

Fig. 19. Organization of the synapse block.

its maximum or minimum. If $\mathrm{A}$ is increased, $\mathrm{L}$ is reset to the value $\mathrm{L}-2{ }^{*} \mathrm{~L} \_$th, but if it is decreased, then $\mathrm{L}$ is reset to $\mathrm{L}+2 *$ L_th. Figure 18 presents the organization of this learning block.

The parameters that govern the functionality of the learning block are:

- The YD variable has a resolution of 6 bits and the learning variable $(\mathrm{L})$ of 8 bits. The activation variable (A) can have four states.

- The time constant for the variable YD is $\tau=20$.

- L_th $=[-128,127]$

To improve the sensitivity of the block for long time difference spikes, the time constant for the variable $\mathrm{L}$ is 4,000 , but it can change depending on the size of the network where the neuron works.

When there are spikes in the actual neuron after the spikes in the projecting neuron, the value of $L$ increases, and the value of $A$ also increases, so the synapse becomes more active.

The goal of the synapse block is to set the value of $J$ (the input value added to the membrane) and it depends on four factors: the synapse activation level (A), the spikes of the projecting neuron $\left(s_{j}\right)$ and the type of the actual neuron and the projecting neuron $\left(r_{i}\right.$ and $\left.r_{j}\right)$.

For each synapse a certain weight is set. This weight is multiplied by the activation variable (A). For this purpose, a shift register is used, so when $A=0$, the weight becomes 0 , when $A=1$ the weight stays the same, when $A=2$ the weight is multiplied by 2 and when $A=3$ it is multiplied by 4 .

This output weight is added to the decayed value of the output J. But the decay curve depends on the type of the actual and the projecting neurons ( $r_{i}$ and $\left.r_{j}\right)$.

There are two possible types for each neuron, excitatory and inhibitory, so we should obtain four possible values for the time constant which will decrease the addition. But, when both neurons are inhibitory, the weight of the synapse is always 0 , so the $J$ value is also always 0 and therefore it is nonsense to decrease it. For this reason, there are only three possible decay time constants.

Table 3. Time constants for different synapse types

\begin{tabular}{lll}
\hline $\begin{array}{l}\text { Time constant } \\
(\tau)\end{array}$ & $\begin{array}{l}\text { Projecting neuron } \\
\text { type }\left(\mathrm{r}_{\mathrm{j}}\right)\end{array}$ & $\begin{array}{l}\text { Actual neuron } \\
\text { type }\left(\mathrm{r}_{\mathrm{i}}\right)\end{array}$ \\
\hline 20 & 0 & 0 \\
0 & 0 & 1 \\
3 & 1 & 0 \\
0 & 1 & 1 \\
\hline
\end{tabular}

In this table $r=0$ means an excitatory neuron, while $r=1$ indicates an inhibitory neuron.

The three time constants are multiplexed, and the multiplexer is controlled by the types of neurons $\left(\mathrm{r}_{\mathrm{i}}, \mathrm{r}_{\mathrm{j}}\right)$. The multiplexer output controls the decay block, and finally we obtain the J value at the output of this decay block. Figure 19 shows the organization of the synapse block.

The parameters that govern the functionality of the synapse block are:

- The internal resolution of the block is 10 bits. But the output resolution is 8 bits, because the internal value of $J$ is divided by 4 to keep the correct scaling.

- The time constants used by this block are presented in table 3 .

The high resolution needed for the variables, as well as the number of operations to be performed may pose a serious limitation for the final implementation. Therefore, the first step in the physical realization of the model consisted of an evaluation of the minimum resolution to be used in the neuron data path.

In a first attempt the resolution of the parameters was reduced by two bits and some values and time constants were changed to keep the correct scaling. Table 4 shows the new values of the internal parameters after this optimization process. The final organization resulting from this optimization process is depicted in figure 20.

Due to the complexity of the design, the simplification of the model is very important to avoid redundancy or to use just the necessary components. For this reason, a further simplification of all the building blocks that constitute the model has been performed [5].

Once the model has been optimized it has been physically translated into the molecules that constitute the basic building blocks of the organic subsystem of the POEtic tissue. Figure 21 shows this physical realization.

The molecule organization shown in figure 21 corresponds to the actual structure of the organic subsystem present in the POEtic tissue, which is arranged as an $8 \times 18$ array of molecules. 
Table 4. Resolution of the parameters for an optimised implementation

\begin{tabular}{ll}
\hline Parameter & New value \\
\hline Membrane resolution & 10 \\
Threshold & +160 \\
Input $(\mathrm{J})$ resolution & 6 \\
Weights & {$[0: 8],[64: 128],[128: 256],[0: 0]$} \\
YD resolution & 4 \\
L resolution & 6 \\
Membrane decay time constant & 20 \\
YD decay time constant & 20 \\
L decay time constant & 4000 \\
J decay time constants $(00,01,10,11)$ & $20,0,3,0$ (keep the same values) \\
\hline
\end{tabular}

After designing the neuron model the VHDL models developed for the POEtic tissue have been configured and simulated to validate its functionality.

After this validation stage the strategy for the simulation of large-sale SNN models has been considered. Since in its actual implementation the POEtic chip only allows for the implementation of a single neuron and the current number of POEtic chips is far less than 10,000 , it will be nec- essary to use a smaller array of POEtic chips whose functionality should be time multiplexed in order to emulate the whole network.

This means that every POEtic chip should be able to manage a local memory in charge of storing the weights and learning variables corresponding to the different neurons it is emulating in time.

A 16-neuron network organized as a $4 \times 4$ array has been constructed using this principle. This would permit the emulation of a 10,000-neuron network in 625 multiplexing cycles. Bearing in mind that each neuron is able to complete a single cycle in 150 clock cycles, this means that the minimum clock frequency required to handle input stimuli in real time (i.e., to process visual input stimuli at 50 frames/s) is around $5 \mathrm{MHz}$, far less than the actual clock frequency achievable by the organic subsystem of the POEtic tissue.

The visual stimuli will come from an OmniVision OV5017 monochrome $384 \times$ 288 CMOS digital camera. Specific VHDL and $\mathrm{C}$ code have been developed in order to manage the digital images coming from the camera. To test the application, artificial image sequences have been generated on a display and then captured by the camera for its processing by the network.

\section{Conclusions}

In this paper we have presented a new family of programmable integrated electronic systems, called POEtic, that include features derived from some of the proper-

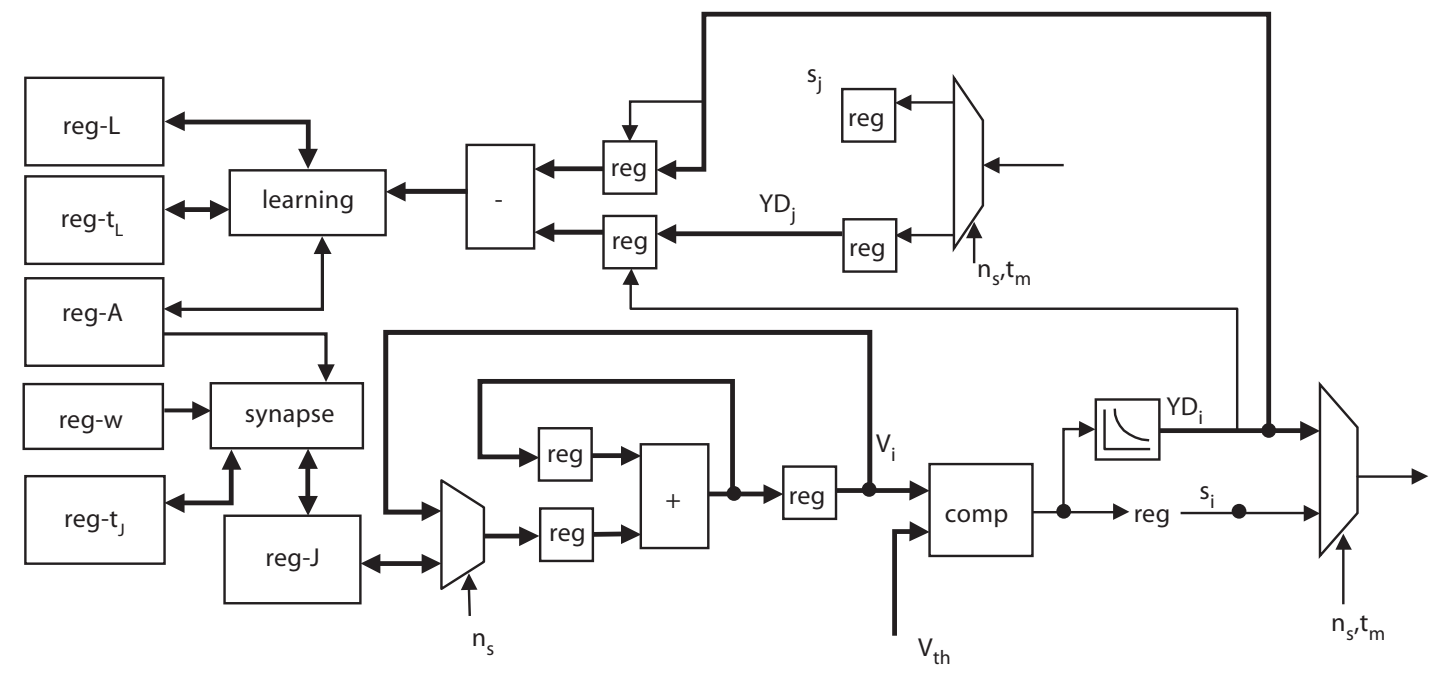

Fig. 20. Block diagram for the serial implementation of the neuron model. 


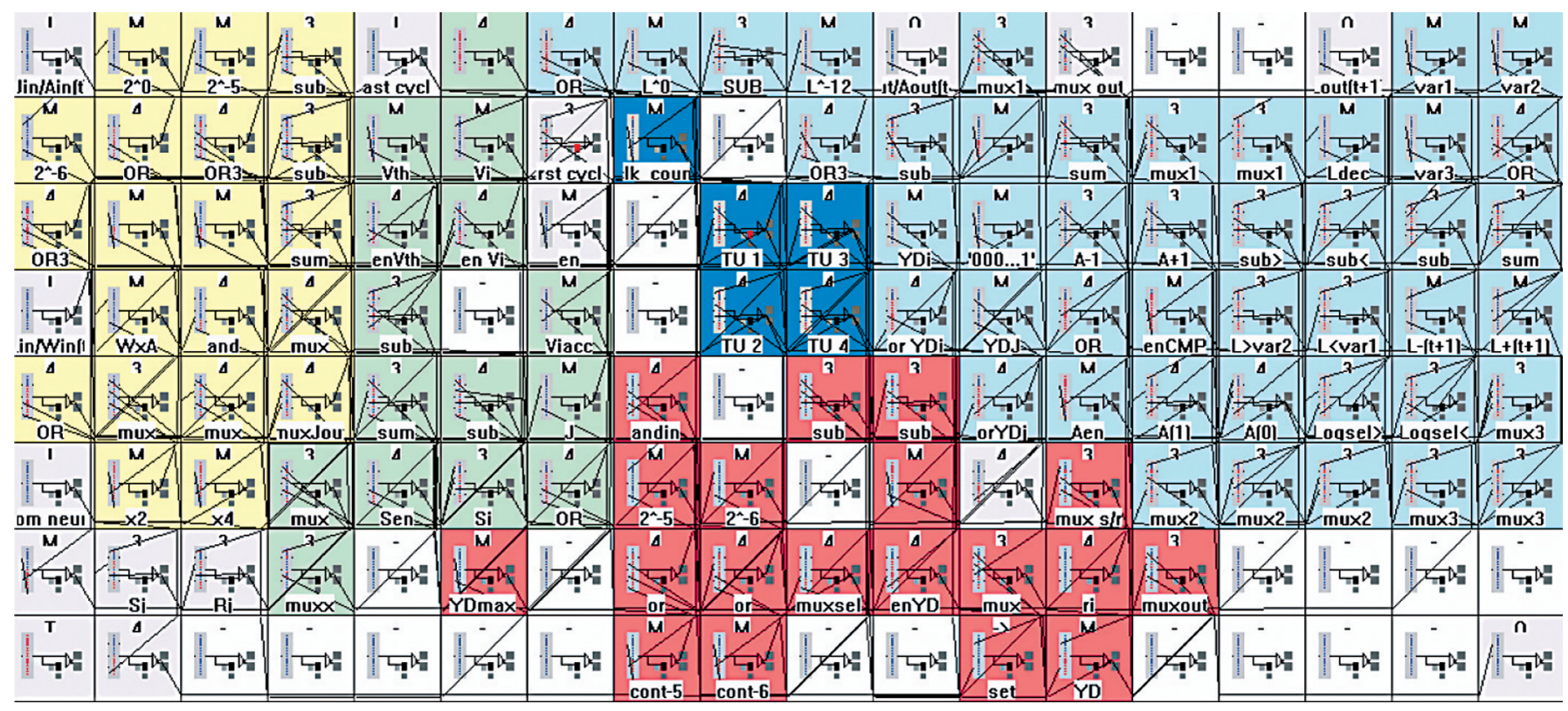

Fig. 21. Molecule level implementation of the neuron model.

ties present in living beings, like evolution, development, self-repair, self-replication and learning.

The combination of partial and total dynamic reconfiguration, as well as the self-configuration and dynamic routing capabilities make these devices an ideal candidate for the efficient implementation of bio-inspired artefacts.

After describing in detail the different building blocks that constitute the tissue, an implementation approach for the emulation of large-scale spiking neural network models has been presented. The results derived from this implementation demonstrate that an electronic tissue built around these devices will permit the realtime emulation of this kind of models, thus serving as an excellent development and experimentation instrument for neuroscientists.

After receiving the first POEtic chips specific development boards have been constructed to develop applications to be solved using the bio-inspired features offered by the tissue.

\section{Acknowledgements}

The work presented in this paper has been funded by the grant IST-2000-28027 (POEtic) of the European Union (FET Proactive Initiative on Neuroinformatics for Living Artefacts) and by grant OFES 00.0529-2 of the Swiss Government. The information provided is the sole responsibility of the authors and does not reflect the European Union's opinion. The European Union is not responsible for any use that might be made of data appearing in this publication.

\section{References}

1 ARM: AMBA Specification, rev 2.0. Advanced RISC Machines Ltd (arm). http://www.arm.com/armtech/ amba_spec, 1999.

2 Dijkstra EW: A note on two problems in connexion with graphs. Numerische Math 1959; 1:269-271.

3 Moreno Arostegui JM, Sanchez E, Cabestany J: An insystem routing strategy for evolvable hardware programmable platforms; in Proc 3rd NASA/DoD Workshop on Evolvable Hardware. IEEE Computer Society Press, 2001, pp 157-166.

4 Eriksson J, Torres 0, Mitchell A, Tucker G, Lindsay K, Halliday D, Rosenberg J, Moreno JM, Villa AEP: Spiking neural networks for reconfigurable POEtic tissue; in Tyrrell AM, Haddow PC, Torresen J (eds): Evolvable Systems: from Biology to Hardware. Berlin, Springer, 2003, pp 165-173.

5 Torres 0, Eriksson J, Moreno JM, Villa A: Hardware optimization and serial implementation of a novel spiking neuron model for the POEtic tissue. Biosystems 2004; 76: 201-208. 